\title{
Die internationalen NRO in Genf
}

Yanik Marguerat

\section{OpenEdition \\ Journals}

Electronic version

URL: http://journals.openedition.org/sjep/497

DOI: $10.4000 /$ sjep.497

ISSN: 1663-9677

\section{Publisher}

Institut de hautes études internationales et du développement

\section{Printed version}

Date of publication: 1 novembre 2004

Number of pages: $35-50$

ISBN: 2-88247-057-6

ISSN: $1660-5926$

\section{Electronic reference}

Yanik Marguerat, "Die internationalen NRO in Genf », Schweizerisches Jahrbuch für Entwicklungspolitik [Online], 23-2 | 2004, Online erschienen am: 08 Juni 2010, abgerufen am 08 September 2020. URL : http://journals.openedition.org/sjep/497 ; DOI : https://doi.org/10.4000/sjep.497 


\title{
Die internationalen NRO in Genf
}

\author{
Yanik Marguerat*
}

\section{Einführung}

Die internationalen Nichtregierungsorganisationen (INRO) nehmen seit den frühen 90er Jahren in den internationalen Verhandlungen einen bedeutenden Platz ein. "Genf" sche Sitz der Vereinten Nationen in Genf hat viele INRO veranlasst, sich in der Calvinstadt anzusiedeln.

Damit bilden die INRO im komplexen Bauwerk des internationalen Genfs einen wichtigen, aber wenig bekannten Baustein. Die vorliegende Studie will einen Überblick über die Welt der INRO in der Region Genf vermitteln und bedient sich dazu einer Vielzahl von Daten, die in einer relationalen Datenbank erfasst und ausgewertet wurden. Als Einführung wird die Welt der INRO beschrieben; der Akzent liegt dabei auf der Struktur, der Funktionsweise, den Gruppierungen in Netzwerken und auf den Problemen bei der Arbeit der INRO.

Anschliessend werden die Auswirkung und die Rolle der INRO für das internationale Genf sowie die Empfangsstrukturen und -politik der Schweizer Behörden beschrieben.

\section{Überblick über die internationalen NRO}

\section{Gruppierung der internationalen NRO um Exzellenzpole}

Für die Zwecke der Untersuchung wurden jeder erfassten INRO maximal zwei prioritäre Tätigkeitsbereiche zugewiesen. Diese Zuordnung erfolgte gemäss dem von der INRO angegebenem Aktivitätsbereich und der Zielanalyse. Anschliessend wurden die (insgesamt 54) verschiedenen Bereiche um 17 Exzellenzpole gruppiert (siehe Tabelle 1), in die sich die INRO klassifizieren lassen².

* Kommunikationsbeauftragter bei der Fédération genevoise de coopération.

Y. Marguerat beteiligte sich an der Studie über die internationalen NRO in Genf, die Gegenstand des vorliegenden Artikels ist. Die Studie wurde im Jahr 2003 im Auftrag des NRO-Dienstes der DEZA von einem von Gérard Perroulaz koordinierten Forscherteam des iuéd durchgeführt. Die Studie verfolgte ein doppeltes Ziel: eine strukturierte und detaillierte Bestandsaufnahme der Welt der INRO in der Region Genf durchzuführen und anschliessend einige Optionen zu Interaktionen zwischen den INRO und dem Bund vorzustellen. Der vorliegende Artikel beschränkt sich auf den ersten Teil. Er vermittelt einen allgemeinen Überblick und präsentiert die Analyse der erhobenen Daten sowie die Rolle der INRO im internationalen Genf. Die vollständige Forschungsarbeit ist bei Gérard Perroulaz unter die e-Mailadresse < gerard.perroulaz@iued.unige.ch> bestellbar .

1 „Genf“ in Anführungszeichen bezieht sich auf die Region Genf, wie sie für die vorliegende Untersuchung definiert wurde. Dazu gehören auch die Kantone Waadt und Wallis sowie das grenznahe Frankreich. Genf ohne Anführungszeichen bezieht sich auf Genf im engeren Sinne (Stadt und Kanton).

2 Eine NRO kann höchstens zwei Polen bzw. maximal zwei verschiedenen Bereichen innerhalb desselben Pols angehören. 


\section{Methodik der Studie}

Für die Standortwahl "Genf" ist für die meisten internationalen NRO die Präsenz des Systems der Vereinten Nationen ein bestimmender Faktor. Internationale NRO werden definiert als Vereinigungen/Stiftungena, die bei einem UNO-Organ einen beratenden oder Beobachterstatus besitzen bzw. von diesen Organen auf der Liste der INRO aufgeführt sind, mit denen sie in Verbindung stehen, und in mindestens zwei Ländern tätig sind. Die Liste umfasst auch die NRO, welche als Fazilitatoren des internationalen Genfs arbeiten.

Auf der Basis der verschiedenen erfassten Listen wurde ein Fragebogen an 420 INRO versandt. Die Zahl der INRO in der Schweiz wird auf 314 geschätzt, davon 276 in "Genf". Ausserdem wurden im Rahmen der Studie zahlreiche Gespräche mit INRO und mit den entsprechenden Kontaktpersonen der Kommunal-, Kantons- und Bundesverwaltungen für die INRO durchgeführt.

a Eine NRO kann in der Schweiz entweder als Verein gemäss Artikel $60 \mathrm{ff}$ des Schweizerischen Zivilgesetzbuches oder als Stiftung gemäss Artikel $80 \mathrm{ff}$ des Schweizerischen Zivilgesetzbuches frei gegründet werden.

Tabelle 1: Exzellenzpole im internationalen Genf, Anzahl der INRO

\begin{tabular}{|c|c|c|c|c|c|}
\hline Pol & $\begin{array}{r}\text { Anzahl } \\
\text { INRO } \\
\end{array}$ & $\ln \%{ }^{a}$ & Pol & $\begin{array}{r}\text { Anzahl } \\
\text { INRO } \\
\end{array}$ & $\ln \%^{a}$ \\
\hline 1. Hilfe & 44 & 11 & 10. Autochthone Völker & 5 & 1 \\
\hline 2. Umwelt & 18 & 4 & 11. Erziehung & 35 & 8 \\
\hline \multirow{2}{*}{$\begin{array}{l}\text { 3. Entwicklungsstudien, } \\
\text { internationale Beziehungen }\end{array}$} & \multirow{2}{*}{13} & \multirow{2}{*}{3} & 12. Religion, Spiritualität, Kultur & 40 & 10 \\
\hline & & & 13. Kunst, Freizeit, Sport & 10 & 2 \\
\hline 4. Recht & 14 & 3 & 14. Vereinte Nationen, Empfang und & & \\
\hline 5 Menschenrechte & 55 & 13 & Förderung der Arbeit der NRO & 21 & 5 \\
\hline 6. Gender & 30 & 7 & 15. Wirtschaft & 26 & 6 \\
\hline 7. Information & 8 & 2 & 16. Handel & 14 & 3 \\
\hline 8. Gesundheit & 41 & 10 & 17. Gewerkschaftsarbeit & 17 & 4 \\
\hline 9. Frieden & 26 & 6 & & & \\
\hline Insgesamt & & & & 417 & 100 \\
\hline
\end{tabular}

a Der Prozentanteil errechnet sich nach der Gesamtzahl der INRO in allen Exzellenzpolen (417), welche höher ist als die Gesamtzahl der INRO (314), da eine INRO maximal zweimal in der Liste gezählt werden kann.

Am meisten Vereinigungen vereint der Pol der Menschenrechte. Das erklärt sich direkt aus der Präsenz des Hochkommissariats für Menschenrechte in Genf. Es wäre denkbar gewesen, dass die Ankunft der Welthandelsorganisation (WTO) zahlreiche INRO anziehen würde; laut den Ergebnissen der Studie sind diesem Pol jedoch in absoluten Zahlen nur wenige Vereinigungen zuzuordnen. Dagegen ist interessanterweise festzustellen, dass eine bedeutende Organisation wie Oxfam International vor kurzem beschlossen hat, in Genf ein kleines Vertretungsbüro zu eröffnen, um die Verhandlungen in der WTO mitzuverfolgen; nach ihrer Auffassung soll das Lobbying offenbar bei den Delegierten vor Ort und nicht vom Londoner Sitz aus betrieben werden.

Auch der Exzellenzpol Frieden ist sehr dynamisch. Das Vorhaben des Bundes (Eidgenössisches Departement für Verteidigung, Bevölkerungsschutz und Sport, VBS), vor dem Zeithorizont 2006-2007 das „Maison de la paix“ (Haus des Friedens) zu bauen, das bestimmte INRO, die drei vom Bund finanzierten sicherheitspolitischen Zentren, das Institut universitaire de hautes études internationales und das iuéd unter einem Dach vereinen könnte, würde klare und deutliche Signale des schweizerischen Engagements für diesen „Nischenmarkt“ aussenden und der Devise „Genève, ville de paix“ (Genf, Stadt des Friedens) Geltung 
verschaffen. Die Organisationen dieses Bereichs, welche im Rahmen der Studie kontaktiert wurden, zeigten Interesse am Vorhaben für ein Haus des Friedens, am Netzwerk und an den vielfältigen Synergien, die sich daraus ergeben könnten. Daher ist zu hoffen, dass der Bund den Bau des „Maison de la paix“ unterstützen und das gleiche Prinzip auf andere Exzellenzzentren anwenden wird, für die Genf ideale Voraussetzungen bietet, z.B. im Bereich der autochthonen Völker. Genf könnte zusammen mit dem Bund die Dynamik des Exzellenzpols nutzen und die Ansiedlung der Botschaft der autochthonen Völker - ein Projekt, das die Karten des internationalen Genfs um einen weiteren Trumpf bereichern würde - nachdrücklich fördern.

In der Darstellung der Welt der INRO wurden ausserdem verschiedene Ebenen der schweizerischen Verwaltung (Bund, Kanton Genf, Stadt Genf) und Instanzen des UN-Systems in die Analyse der Pole einbezogen. Sofern es relevant und kohärent erschien, wurde jeder Bundesstelle bzw. allen Agenturen, Programmen oder Fonds der UNO (mit Sitz oder Regionalbüro in Genf) ein oder mehrere Tätigkeitsbereiche zugeordnet. So ist für jeden Exzellenzpol auf den ersten Blick ersichtlich, welche öffentlichen Verwaltungsorgane betroffen sind, welche UNO-Organe relevant sind und welche INRO für den fraglichen Pol eine Rolle spielen. Die INRO werden entweder konkret in einem gemeinsamen Raum („Haus“) vereint oder arbeiten in einem „Netz“ zusammen. Die in der Studie verfolgte Vorgehensweise wird in Grafik 1 zusammengefasst.

\section{Grafik 1: Ansatz nach Exzellenzpolen}

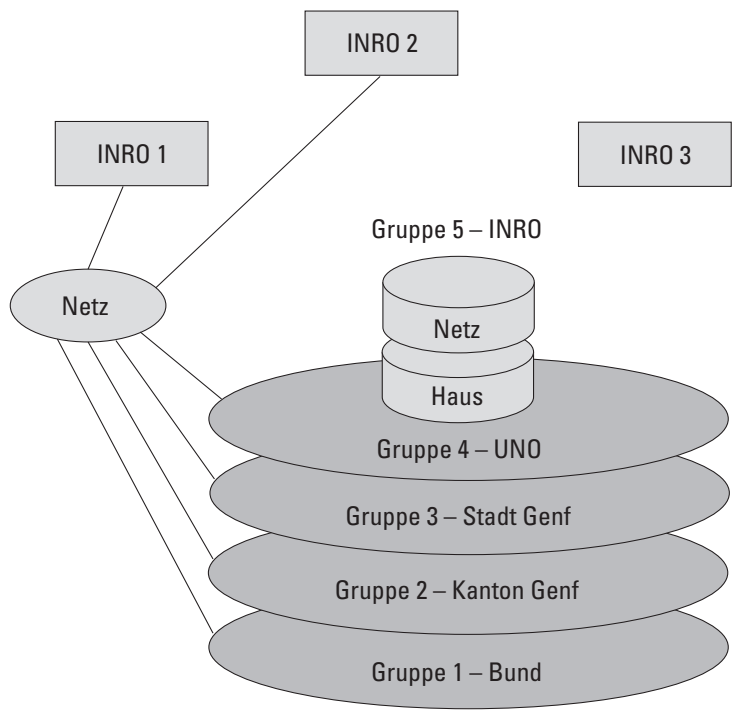

Das Analyseraster in der Klassifizierung nach Polen lässt sich noch verbessern; es sollte als erste Version eines ausbaufähigen Instruments gesehen werden, das noch verfeinert und ergänzt werden kann. Um die Möglichkeiten dieses Analyseinstrumentes zu verdeutlichen, wurde der Exzellenzpol Umwelt eingehender behandelt und im Rahmen einer Fallstudie grafisch dargestellt. 


\section{Fallstudie „Umwelt”}

Grafik 2: Exzellenzpol Umwelt:

Detaillierte Illustration der Methodik der Studie anhand des Fallbeispiels Umwelt

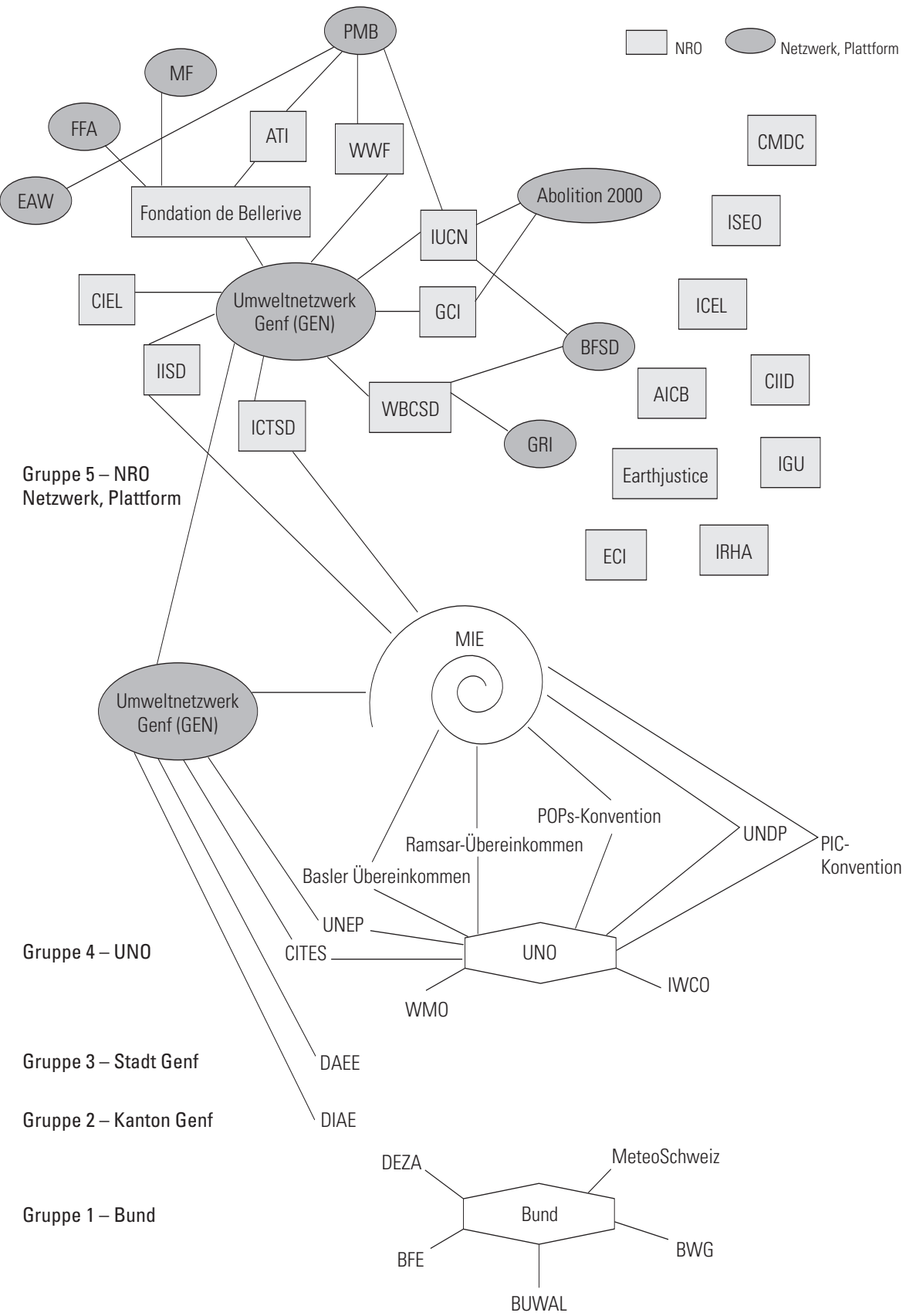


AICB

ATI

Basler Übereinkommen

BFE

BFSD

BUWAL

BWG

CIEL

CIID

CITES

CMDC

DAEE

DEZA

DIAE

EAW

ECI

FFA

$\mathrm{GCl}$

GEN

GRI

ICEL

ICTSD

IGU

IISD

IRHA

ISEO

IUCN

IWCO

MeteoSchweiz

MF

MIE

PIC-Konvention

PMB

POPs-Konvention

Ramsar-Übereinkommen

UNDP

UNEP

WBCSD

WMO

WWF
International Association against Noise

Amis de la terre international

Basler Übereinkommen über die Kontrolle der grenzüberschreitenden Verbringung gefährlicher Abfälle und ihrer Entsorgung

Bundesamt für Energie (UVEK - Departement für Umwelt, Verkehr, Energie und Kommunikation)

Bellagio Forum for Sustainable Development

Bundesamt für Umwelt, Wald und Landschaft (UVEK)

Bundesamt für Wasser und Geologie (UVEK)

Center for International Environmental Law

Internationale Kommission für Bewässerung und Entwässerung

Übereinkommen über den internationalen Handel mit gefährdeten Arten frei lebender Tiere und Pflanzen

Weltkreis des Konsens

Departement für soziale Angelegenheiten, Schulen und Umwelt der Stadt Genf

Direktion für Entwicklung und Zusammenarbeit (EDA - Eidgenössisches Departement für auswärtige Angelegenheiten)

Departement des Inneren, der Landwirtschaft und Umwelt (Kanton Genf)

Eurogroup for Animal Welfare

Earth Council Institute

Fur Free Alliance,

Green Cross International

Umweltnetz Genf

Global Reporting Initiative

International Council of Environmental Law

International Centre for Trade and Sustainable Development

International Gas Union

International Institute for Sustainable Development

International Rainwater Harvesting Alliance

International Sustainable Energy Association

World Conservation Union

Independent World Commission on the Oceans

Bundesamt für Meteorologie und Klimatologie, MeteoSchweiz (EDI - Eidgenössisches Departement des Inneren)

Mountain Forum

Internationales Haus der Umwelt

Übereinkommen über das Verfahren der vorherigen Zustimmung nach Inkenntnissetzung für bestimmte gefährliche Chemikalien sowie Pestizide im internationalen Handel

Pro Mont-Blanc

Stockholmer Übereinkommen über persistente organische Schadstoffe

Übereinkommen der UNESCO über Feuchtgebiete, insbesondere als Lebensraum für Wasser- und Watvögel, von internationaler Bedeutung

Entwicklungsprogramm der Vereinten Nationen

Umweltprogramm der Vereinten Nationen

World Business Council for Sustainable Development

Weltorganisation für Meteorologie

World Wide Fund for Nature

Der Umweltpol ist sehr klar strukturiert. Das erklärt sich aus der Präsenz des „Maison internationale de l'environnement“ (Internationales Haus der Umwelt) und des „Réseau environnement Genève GEN“ (Umweltnetz Genf), welche die INRO mit den UNO-Organisationen sowie den Dienststellen des Kantons, der Stadt Genf und indirekt mit denjenigen des Bundes verbinden. 
Mitglieder des GEN sind INRO und internationale Organisationen (IO) der Vereinten Nationen sowie internationale Umweltabkommen. Die Mehrheit dieser Organisationen (NRO und IO) haben im „Maison internationale de l'environnement" ein Büro und teilen sich dort einen gemeinsamen Raum.

Das „Réseau environnement Genève“ entstand gleichzeitig mit dem Bau des „Maison internationale de l'environnement“ (MIE) ${ }^{3}$ als Ergebnis einer vom Bundesamt für Umwelt, Wald und Landschaft (BUWAL) angeforderten Machbarkeitsstudie. Heute zählt das Netz rund vierzig Mitglieder. Ein Koordinator übernimmt die Federführung für die Arbeit dieser Struktur.

Zum gleichen Zeitpunkt, als das Internationale Haus der Umwelt in Genf gegründet wurde, wanderten drei wichtige Sekretariate von auf der Rio-Konferenz ausgehandelten Abkommen ins Ausland $\mathrm{ab}^{4}$. In diesem Zusammenhang ist interessanterweise festzustellen, dass der Bund heute die Präsenz des Netzes und des Internationalen Hauses der Umwelt in „Genf“ als schlagkräftiges Argument ins Feld führt, um Übereinkommen wie die Konvention von Stockholm (POPS-Konvention) $)^{5}$ und die Konvention von Rotterdam, deren gemeinsames Sekretariat zwischen „Genf“" (UN-Umweltprogramm) und Rom (FAO) aufgeteilt werden soll, vom Standort Genf zu überzeugen.

Die Synergien, die sich teilweise bereits aus dem Netz ergeben, erhalten durch das Zusammenspiel mit der gemeinsamen Unterbringung im Haus der Umwelt eine zusätzliche Dynamik. Die Organisationen verfügen über ein gemeinsames Dokumentationszentrum, eine Cafeteria und gemeinsame Konferenzsäle. So lassen sich die Betriebskosten für die INRO gering halten. Ausserdem erleichtert die unmittelbare Nachbarschaft im gleichen Gebäude den Austausch unter den verschiedenen Akteuren.

Das zweite Internationale Haus der Umwelt (MIE 2) wurde am 4. Juni 2003 anlässlich des Internationalen Umwelttags eingeweiht. Es wurde dank der Initiative des Bundes, des Kantons und der Stadt Genf gebaut. Das MIE 2 bildet eine Ergänzung zum vor fünf Jahren gegründeten Haus der Umwelt und wird in erster Linie die nichtstaatlichen Umweltorganisationen beherbergen.

Es wird interessant sein, zu sehen, welchen Weg die Bundesbehörden beim Aufbau und bei der Organisation des Hauses des Friedens einschlagen werden, das wie erwähnt für den Zeitraum 2006-2007 geplant ist.

3 Das im September 1999 eingeweihte „Maison internationale de l'environnement“ wurde den Organisationen, die in Genf im Bereich Umwelt und nachhaltige Entwicklung tätig sind, zur Verfügung gestellt. Der Bund ist Eigentümer der von der FIPOI (Fondation des immeubles pour les organisations internationales, Immobilienstiftung für die internationalen Organisationen) verwalteten Liegenschaft. Die darin untergebrachten internationalen Umweltorganisationen bezahlen keine Miete, bestreiten aber die Unterhaltskosten sowie die üblichen Nebenkosten.

4 Das Sekretariat der Biodiversitätskonvention ist nach Montreal umgezogen, die Sekretariate der Übereinkommen über den Klimawandel und über die Wüstenbildung sind nach Bonn umgesiedelt.

5 In der Genfer Werbebroschüre für die Aufnahme des Sitzes des ständigen Sekretariats des Übereinkommens von Stockholm werden die Vorzüge des Internationalen Hauses der Umwelt und des Umweltnetzes Genf unterstrichen. Siehe Bundesamt für Umwelt, Wald und Landschaft (BUWAL), Geneva!, Bern, Juni 2002. 


\section{Kommentar zur Datensynthese}

Die Antworten der INRO auf den Fragebogen erlauben mehrere Feststellungen zur Welt der internationalen Nichtregierungsorganisationen. Die Anzahl der INRO, die in „Genf“ gegründet wurden oder ein Büro eröffnet haben, nimmt zu. Besonders im Zeitraum 1990-2003 wurde ein starker Zuwachs verzeichnet: Die Zahl der neu gegründeten INRO ist von 25 auf 56 angestiegen und hat sich im Vergleich zum vorhergehenden Jahrzehnt mehr als verdoppelt. Am dynamischsten waren in den 90er Jahren die beiden Exzellenzpole Menschenrechte und Gesundheit, auf die je neun neue INRO entfielen.

Die INRO, welche die Frage zu den personellen Ressourcen beantworteten, beschäftigen insgesamt 2394 Angestellte und 1112 Freiwillige (Total 3506 Personen). Die Auffächerung der Beschäftigten nach Polen zeigt, dass ein grosser Teil der Angestellten auf die Wirtschaft entfällt (425), gefolgt von den Polen Kunst, Freizeit, Sport (330), Umwelt (324) und Information (300). Die drei ersten Pole entsprechen gleichzeitig der INRO-Gruppe mit dem höchsten Betriebshaushalt.

Hinsichtlich der Anzahl der Mitarbeitenden ist das Genfer Büro einer INRO mit einem Kleinunternehmen vergleichbar ${ }^{6}$. 74 Prozent der INRO beschäftigen weniger als zehn Angestellte und 91 Prozent weniger als zehn Freiwillige. 64 Prozent der INRO beschäftigen insgesamt weniger als zehn Personen. Die Gruppierung der INRO nach der Höhe ihres Budgets ergab bei 64 Prozent der Antworten einen Etat von höchstens 1 Million Franken.

Zur Finanzierungsform ist festzustellen, dass 57 Prozent der INRO ihre Mittel zu über drei Vierteln selbst erwirtschaften oder aus privaten Quellen beziehen. Nur 13 Prozent der INRO decken über drei Viertel ihres Bedarfs mit öffentlichen Finanzierungen. Demnach ist ein bedeutender Teil der INRO stärker auf Privat- und Selbstfinanzierung als auf öffentliche Mittel angewiesen.

Nur 43 (28\%) der 153 INRO, die eine Antwort zurücksandten, erwähnten Beihilfen der öffentlichen Behörden der Schweiz. Die Behörden leisten in erster Linie finanzielle Unterstützung; der Bund spielt dabei eine Schlüsselrolle. Daneben leisten die Genfer Kantons- und Kommunalbehörden auch logistische und materielle Hilfe.

Die Institutionen der Vereinten Nationen stehen unter den anderen Akteuren in „Genf“, mit denen die INRO zusammenarbeiten, an erster Stelle, gefolgt von den anderen INRO. Die Zuordnungsstatistik zu den Vereinten Nationen und zu den Unterorganisationen zeigt, dass die Menschenrechtskommission am engsten mit den INRO zusammenarbeitet. Dies lässt sich mit der grossen Zahl der im Menschenrechtsbereich tätigen INRO in Genf erklären.

6 Interessanterweise weisen die INRO im Durchschnitt relative hohe und kaum repräsentative Betriebshaushalte und bezahlte Stellen auf. Eine INRO verfügt durchschnittlich über einen Betriebshaushalt von 4,1 Millionen Franken und beschäftigt 17 Angestellte und 8 Freiwillige (insgesamt 25 Personen). Diese relativ hohen Zahlen erklären sich direkt aus den Eckdaten der 20 grössten INRO, deren Budgets und Beschäftigtenzahlen deutlich über jenen der meisten anderen INRO liegen. Aus diesem Grund sind die durchschnittlichen Kennzahlen einer INRO in Genf wenig repräsentativ. 


\section{Plattformen und Netzwerke der internationalen $\mathrm{NRO}^{7}$}

Laut Angaben der kontaktierten INRO gewinnt der Aspekt der „Vernetzung“ zunehmend an Bedeutung. Die INRO setzen sich vermehrt mit komplexen Themen auseinander. Ausserdem handelt es sich bei den meisten erfassten INRO wie erwähnt um kleinere Organisationen mit begrenzten Mitteln. Aus diesem Grund haben einige INRO ihre Ressourcen und Bemühungen vereint, um effizienter zu arbeiten und mit vereinten Kräften Aufgaben zu verwirklichen, die eine INRO im Alleingang nicht bewältigen könnte.

Mit dem an die INRO gesandten Fragebogen konnten zudem die Netzwerke und Plattformen identifiziert werden, über welche die INRO zusammenarbeiten und Informationen austauschen. Dabei fällt als erstes ins Auge, dass bestimmte INRO in der Datenbank von ihrer Struktur her bereits INRO-Netze oder -Plattformen bilden. Die beiden mitgliederstärksten Plattformen sind die CONRO (Conférence des ONG ayant le statut consultatif avec les Nations unies, Konferenz der NRO mit Beraterstatus bei den Vereinten Nationen) und die FIIG (Fédération des institutions internationales semi-officielles et privées établies à Genève, Vereinigung der in Genf niedergelassenen halbamtlichen und privaten internationalen Institutionen). Zahlreiche der in der Datenbank erfassten Organisationen gehören diesen beiden Strukturen mit Sitz in Genf an.

Neben diesen beiden Strukturen nannten die INRO im Fragebogen 73 weitere Netze, denen mindestens eine Vereinigung in der Datenbank als Mitglied angehört.

Die Analyse der verschiedenen Netze und deren Mitglieder zeigte, dass sich in den meisten Netzen auch INRO aus dem Bereich „Religion, Spiritualität, Kultur" befanden. Die Tätigkeiten der INRO dieses Pols sind äusserst vielfältig und berühren mehrere Bereiche.

\section{Vor- und Nachteile der vernetzten Arbeitsweise}

Die Hauptvorteile der vernetzten Arbeitsweise sind nach Auffassung der befragten Organisationen die Möglichkeiten, Informationen auszutauschen, Synergien $\mathrm{zu}$ erschliessen und in bestimmten Bereichen gemeinsame Kompetenz- und Expertisepools aufzubauen sowie die Arbeit zu teilen. Diese Vorteile ermöglichen den als Netzwerke gegliederten INRO, effizienter zu arbeiten, in einer bestimmten Thematik grösseren Einfluss auszuüben und die Zielsetzungen rascher zu erreichen. Ein weiterer Vorteil besteht nach Ansicht der befragten Organisationen darin, Doppelspurigkeiten zu vermeiden, ihre Positionen einander gegenüberzustellen und die Diskussion und Reflexion in bestimmten Themenbereichen auszuweiten.

Koordination und Konsultation sind zwei Schlüsselfaktoren für die effiziente Nutzung eines Netzes: Aus den Interviews ging klar hervor, dass Netze einen erheblichen Aufwand an Zeit und Ressourcen erfordern. Wie bereits festgestellt, sind die meisten INRO kleinere Organisationen mit sehr knappen finanziellen und personellen Ressourcen. Bisweilen steht die für eine aktive Mitwirkung in Netzwerken erforderliche Investition in keinem Verhältnis zum erwarteten

Dieses Unterkapitel beruht auf den Daten, die mit dem Fragebogen und in Gesprächen mit den NRO erhoben wurden. 
Gewinn. Bestimmte Organisationen hatten Schwierigkeiten bei der Suche nach geeigneten Arbeitspartnern mit ähnlichen Zielsetzungen; das erklärt sich aus der mangelhaften Kenntnis der Welt der INRO am Standort "Genf" ${ }^{\text {" }}$. Als weitere Probleme nennen die - in der Regel sehr auf ihre Unabhängigkeit bedachten INRO den Verlust des Profils, der Identität oder der Autonomie.

Aus den Gesprächen geht zudem hervor, dass die befragten INRO die Zusammenarbeit in informellen und ad hoc-Strukturen bzw. einer Mischung aus formellen und informellen Netzen vorziehen'. Generell setzen die INRO in zentralen, für ihre Arbeit grundlegenden Fragen auf flexible und informelle Zusammenschlüsse, um die erwünschten Ergebnisse schneller zu erzielen. Formelle Strukturen gelten jedoch keinesfalls als nutzlos, im Gegenteil: Zahlreiche INRO halten deren Repräsentationsarbeit bei der UNO durchaus für sinnvoll, beurteilen aber den Austausch und die Synergien, die sich aus solchen Strukturen ergeben, als relativ unrelevant für die Alltagsarbeit ${ }^{10}$.

\section{Schwierigkeiten der internationalen NRO bei der Durchführung ihrer Tätigkeiten}

Die Interviews mit den INRO und die anhand der Fragebögen erhobenen Daten liessen verschiedene Schwierigkeiten ersehen, auf welche die INRO in ihrer Arbeit stossen.

Vor dem aktuellen internationalen Kontext kommt häufig das Problem der Konkurrenz unter den INRO zur Sprache, die sich in der eindrucksvollen Zunahme der Anzahl der INRO äussert. Bisweilen herrscht unter den Vereinigungen ausgeprägtes Rivalitätsdenken, welches gravierende Probleme aufwerfen kann. Die ungünstige Konjunktur und das derzeitige politische Klima sind der Suche nach neuen öffentlichen Finanzierungsquellen für die Organisationen nicht förderlich, und die begrenzten Ressourcen müssen auf eine ständig wachsende Anzahl von Organisationen verteilt werden.

Die erhobenen Daten zeigen, dass die INRO in „Genf“ zu den Behörden in der Bundeshauptstadt Bern relativ lose und unregelmässige Kontakte haben ${ }^{11}$. Viele

8 Bisweilen sind die Organisationen zwar sehr eng mit Netzen oder Koalitionen verbunden, deren Sekretariate in aller Herren Länder verstreut sind, kennen aber die anderen Organisationen, die in Genf im gleichen Bereich arbeiten, nur oberflächlich. Dies gilt besonders für die jüngst geschaffenen Organisationen (,Ableger“ von grösseren Föderationen mit Sitz im Ausland, wie Oxfam International oder Human Rights Watch).

9 „Formelles Netz“ bezieht sich auf eine ständige Struktur, die ein von einer oder mehreren Personen koordiniertes Sekretariat besitzt und genaue Bedingungen für die Aufnahme und Anerkennung der Mitglieder vorschreibt. „Informelles Netz“ entspricht einem Zusammenschluss mehrerer INRO um ein Thema oder um ein genaues und zeitlich befristetes Arbeitsziel (ohne Sekretariat bzw. formelle Anerkennung der Mitglieder).

10 Das Beispiel der Organisation ICVA (International Council of Voluntary Agencies) ist in vielerlei Hinsicht aufschlussreich: Dieses 1962 in Genf gegründete NRO-Netz umfasste ursprünglich Organisationen aus verschiedensten Bereichen. Im Rahmen einer tief greifenden Umstrukturierung wurde 1997 die Neuausrichtung auf Flüchtlinge und humanitäre Fragen beschlossen (die Belegschaft wurde von 12 auf 4 Personen reduziert). Dabei handelt es sich um ein bezeichnendes Beispiel für die aktuelle Tendenz zur Spezialisierung und zu Strukturen, die sich auf einen engeren Themenkreis konzentrieren.

11 Den erhobenen Daten ist zu entnehmen, dass nur 36 der befragten INRO wöchentliche oder monatliche Kontakte mit dem Bund unterhalten. Dagegen pflegen über 116 INRO gleich häufige Kontakte mit der UNO und ihren Unterorganisationen. 
Organisationen, die ein grösseres Engagement und eine klarere Politik des Bundes für die INRO wünschen, beklagen die gegenseitige Unkenntnis. Sie werfen Bern mangelndes Interesse an der Welt der INRO vor. Andere Organisationen hatten Schwierigkeiten, mit den Bundesbehörden Kontakt aufzunehmen. Diese Situation hat offensichtlich zwei Gründe: Erstens gibt es in Bern keine „einheitliche Anlaufstelle" für die NRO, welche häufig nicht wissen, an wen sie sich wenden müssen. Zweitens beklagen die INRO das Fehlen einer globalen Vision in der Bundesverwaltung, welche tatsächlich weder einen Überblick, noch eine klar definierte Politik in ihrer Aktion für die INRO besitzt. Interessanterweise konnte der Bund im Rahmen der Studie keine umfassenden Statistiken über die Unterstützung Berns an die INRO vorlegen. So mussten die einzelnen Departemente der Bundesverwaltung unter die Lupe genommen und die verschiedenen Beihilfen der Dienste und Stellen addiert werden, um einen Überblick über die Unterstützung des Bundes an die internationalen NRO zu erhalten.

Bestimmte INRO erwähnen bei der Durchführung ihrer Tätigkeiten Schwierigkeiten im Berufs- und Privatleben. In diesem Zusammenhang wurde die Gewährung einer Arbeitsbewilligung oder eines Visums für die Delegierten und das Arbeitsverbot für Ehepartner von ausländischen INRO-Mitarbeitenden häufig erwähnt.

Hinsichtlich der Lebensbedingungen für die Angestellten von INRO warnten zahlreiche Stimmen, dass das Wachstum des internationalen Sektors in "Genf“ die derzeit gut benoteten Verhältnisse und die Qualität der Infrastruktur nicht beeinträchtigen dürfe. Der Zugang zu erschwinglichen internationalen Schulen ist mit ein entscheidender Faktor für die Lebensqualität und sollte nach Auffassung der INRO von den Behörden gefördert werden.

Rolle der internationalen INRO für das internationale Genf Strukturen und Empfangspolitik in "Genf"

Mehrere jüngere Studien enthalten Zahlenmaterial zur Auswirkung des internationalen (staatlichen und nichtstaatlichen) Sektors auf die Genfer Wirtschaft. Auf eine Zusammenfassung der Zahlen wird hier verzichtet ${ }^{12}$. Der Vergleich der verschiedenen Arbeiten wird allerdings durch die je nach Quelle stark variierenden Indikatoren und Kriterien erschwert. Gemäss den im Rahmen dieser Forschungsarbeit erhobenen Daten wird die Anzahl der INRO in der Schweiz auf 314 geschätzt. Das entspricht 2448 bezahlten Stellen und generiert 428 Millionen Schweizer Franken Betriebskosten.

Das vorliegende Kapitel beginnt mit einer kurzen Beschreibung der Empfangsstrukturen und -politik des Kantons Genf für die INRO. Ziel ist es, einen Überblick über die Empfangs- und Förderstrukturen des nichtstaatlichen Sektors und über deren jeweilige Rolle $\mathrm{zu}$ vermitteln. Anschliessend wird die wachsende Rolle der INRO aus der Perspektive der Schweiz und der Vereinten Nationen geschildert. Zum Schluss werden die Vor- und Nachteile des Standorts „Genf“ für die INRO beleuchtet.

12 Siehe Bibliographie am Ende des Artikels. 


\section{Politik der schweizerischen Behörden für die internationalen NRO; vorhandene Empfangsstrukturen}

Das internationale Genf betrifft den Kanton und den Bund gleichermassen. In der Praxis haben sich Bern und Genf indessen darauf geeinigt, dass das Thema Empfang der internationalen Regierungsorganisationen (UNO, IO, ständige Vertretungen, Delegationen) in den Kompetenzbereich Berns fällt, und dass der Kanton Genf für die INRO zuständig ist. Im Jahr 1995 verlangte die Genfer Regierung von Bern eine Entschädigung für die immer wichtigere Rolle des Kantons bei der Förderung und Wahrung des internationalen Genfs. Diese Forderung mündete in die Einsetzung der ,ständigen gemischten Arbeitsgruppe Bund-Kanton Genf über die Prioritäten des internationalen Genfs“, welche unter anderem die möglichen Beihilfen und die punktuelle Unterstützung an „Genf“ sowie die optimalen Empfangsbedingungen für die INRO untersuchen sollte. Die ständige gemischte Arbeitsgruppe formulierte verschiedene Empfehlungen, die zu von Kanton und Bund unterstützten konkreten Aktionen führten ${ }^{13}$.

Als erstes wurde im Jahr 1996 in einer gemeinsamen Initiative des Kantons und des Bundes das Geneva Welcome Center (CWC) und das Amt des NRO-Koordinators eingerichtet, der die NRO über eine Niederlassung in Genf informiert und berät. Im gleichen Jahr setzte der Genfer Staatsrat die vom Protokollchef präsidierte „Commission interdépartementale sur la Genève internationale, CIGI" (Interdepartementale Kommission für das Internationale Genf) ein. Die CIGI prüft die Unterstützungsgesuche der NRO und leistet in verschiedenen Bereichen Beihilfe. Im Jahr 1997 legte die Genfer Kantonsregierung im Sitzungsprotokoll des Staatsrates über die Evaluationskriterien der NRO eindeutig fest, nach welchen Kriterien sich der Kantonsbeitrag an die NRO richtet.

Neben den genannten offiziellen Strukturen spielen weitere NRO und Einrichtungen für die Aufnahme und die Unterstützung der Arbeit der NRO und der nichtstaatlichen Delegierten eine zentrale Rolle. Einige Beispiele werden im Folgenden genannt.

Die Vereinigung Mandat International, welche das „Centre d'accueil pour les délégués des ONG, CADONG“ (Empfangszentrum für NRO-Delegierte) verwaltet, erweist besonders den nichtstaatlichen Delegierten aus Ländern des Südens hilfreiche Dienste: Unterbringung der nichtstaatlichen Delegierten und der Vertreter der autochthonen Völker in Genf, Ausbildung und Beratung der Delegierten. Das DOCIP (Dokumentations-, Forschungs- und Informationszentrum für autochthone Völker) fördert und erleichtert die Arbeit der autochthonen Völker innerhalb des Systems der Vereinten Nationen. Der „International Service for Human Rights“ (Internationaler Dienst für Menschenrechte) bietet den Menschenrechtsaktivisten Unterstützung, Hilfe und Ausbildung für das Lobbying bei den Menschenrechtsstellen der Vereinten Nationen an. Die Vereinigung der in Genf niedergelassenen halbamtlichen und privaten internationalen Institutionen (FIIG) verteidigt und fördert die Interessen der Mitglieder (124 NRO) bei ihren Aktivitäten in Genf. Auch die „Fondation pour Genève“ (Stiftung für Genf) spielt für den Empfang der internationalen Gäste in Genf eine wichtige

13 Siehe den Bericht von Jean-Luc Chopard (Protokollchef, Kanzlei Genf), Genève et les organisations internationales non gouvernementales, Genf, Februar 2002, 7 S. 
Rolle. Sie engagiert sich im Wesentlichen im informellen Rahmen für die Verbesserung der Beziehungen zwischen der internationalen Gemeinschaft und der Genfer Bevölkerung.

Die NRO sind auch im System der Vereinten Nationen in Genf vertreten, wo ihre Interessen in vier Strukturen wahrgenommen werden: erstens im NRO-Verbindungsbüro der Vereinten Nationen, das unter anderem für den Beratungsstatus im Wirtschafts- und Sozialrat ECOSOC zuständig ist; zweitens im NonGovernmental Liaison Service (nichtstaatlicher Verbindungsdienst), einem Interagency-Programm der UNO, das den Dialog erleichtern und die Zusammenarbeit zwischen der UNO und der NRO-Gemeinschaft weltweit fördern soll; drittens im CONRO-Netz (Conférence des ONG ayant le statut consultatif avec les Nations unies, Konferenz der NRO mit Beratungsstatus bei den Vereinten Nationen), einer nichtstaatlichen Vereinigung zur Erleichterung der gemeinsamen Bemühungen der NRO um die Beteiligung an der Arbeit der Vereinten Nationen; viertens im Department of Public Information (DPI, Abteilung Öffentlichkeitsinformation) der UNO mit einem NRO-Dienst, welcher die Verbindung zwischen dem DPI und den NRO gewährleistet.

Aus der kurzen Beschreibung geht hervor, dass "Genf“ über zahlreiche, aber relativ verstreute Empfangsstrukturen verfügt. Die UNO, die Stadt und der Kanton Genf sowie bestimmte Organisationen mobilisieren zwar beträchtliche Ressourcen und Energie, um die Arbeit der INRO zu erleichtern, doch lässt die Koordinierung bisweilen zu wünschen übrig. Der Ausbau der Empfangsstrukturen für den nichtstaatlichen Sektor in „Genf“ hängt direkt mit der steigenden Zahl der INRO und ihrer wachsenden Bedeutung zusammen.

Die INRO stellen für Genf über die genannten ökonomischen Aspekte hinaus einen wichtigen Trumpf dar. Die Rolle der INRO in internationalen Gremien und in der Aussenpolitik der Schweiz wird allmählich besser bekannt. Für ihre Dynamik, ihre Unabhängigkeit, ihre Innovationskraft und die Fähigkeit, sich an verschiedenste Situationen anzupassen, verdienen sie grössere Unterstützung durch die Bundes- und Kantonsbehörden. Bestimmte, auf ihre Unabhängigkeit und ihren Handlungsspielraum bedachte Organisationen möchten verständlicherweise keine finanzielle Hilfe der Behörden in Anspruch nehmen. Die Behörden müssen aber dafür sorgen, dass die Organisationen, ihr Personal und ihre Delegierten gute Rahmenbedingungen für ihre Arbeit vorfinden, beispielsweise mit Verfahrenserleichterungen bei der Visumsgewährung oder mit materieller und logistischer Hilfe für die Anreise und Unterbringung von nichtstaatlichen Delegationen.

Genf besitzt wie erwähnt mehrere Exzellenzpole, z.B. in den Bereichen Menschenrechte, Frieden und Umwelt. Die Behörden sollten ihre Unterstützung dementsprechend ausrichten und gleichzeitig die Bemühungen zur Förderung der Funktionsweise der INRO, welche in den verschiedenen Exzellenzpolen tätig sind, koordinieren und bündeln.

\section{Wachsende Rolle der internationalen NRO aus der Perspektive der Schweiz und der Vereinten Nationen}

Im System der Vereinten Nationen ist gegenwärtig ein tief greifender Überlegungsprozess zum besseren Verständnis und zur Neudefinition der Beziehungen 
zwischen Zivilgesellschaft, Privatsektor und Staaten im Gange. Ein von Generalsekretär Kofi Annan eingesetzter hochrangiger Ausschuss befasst sich mit dieser Frage und bemüht sich mit Blick auf eine ,global governance“, welche die staatlichen und die nichtstaatlichen Akteure umfasst, künftige Wege der möglichen Zusammenarbeit und Interaktion zwischen den Organisationen der Zivilgesellschaft, dem Privatsektor und den Vereinten Nationen zu umreissen ${ }^{14}$. Der Ausschuss unterbreitete dem UNO-Generalsekretär im Mai 2004 einen Bericht mit Empfehlungen. Der ehemalige brasilianische Präsident Henrique Cardoso äusserte sich dazu wie folgt: „Civil society, in its constituent diversity, is a key promoter of multilateralism. Its power is not the authority to decide or to enforce. It is the capacity to argue, to denounce, to propose, to experiment, to innovate, to be exemplary. "15 Die Schlussfolgerungen des Ausschusses und die möglichen Lehren für die Schweizer Behörden werden mit grossem Interesse erwartet ${ }^{16}$.

Die NRO und die Organisationen der Zivilgesellschaft werden in verschiedenen Dokumenten der Bundesverwaltung als nichtstaatliche Akteure beschrieben, welche die weltweit getroffenen politischen Beschlüsse mehr oder weniger stark beeinflussen können. Die Hauptherausforderung besteht heute für den Bund darin, aus der Häufung der nichtstaatlichen Akteure Nutzen zu ziehen und in den multilateralen Verhandlungen den Beitrag der INRO zur Geltung zu bringen. Der Bund zeigt sich in dieser Hinsicht aufgeschlossen. So wurden z.B. NRO-Delegierte in bestimmte Regierungsdelegationen aufgenommen - letztmals in die schweizerische Delegation anlässlich der ersten Phase des Weltgipfels über die Informationsgesellschaft (WSIS) im Dezember 2003 in Genf.

\section{Vor- und Nachteile des Standorts "Genf" für internationale NRO}

In der Folge werden die von den INRO aufgeführten Hauptvorteile genannt: Die INRO halten die Präsenz der UNO, der internationalen Organisationen und der diplomatischen Vertretungen in Genf für sehr wichtig. Der gute Ruf von „Genf" im Ausland, die Neutralität und die Tatsache, dass die Schweiz nicht durch eine koloniale Vergangenheit belastet ist, die geografische Lage der Stadt im Herzen Europas und der Flughafen zählen zu den Faktoren, welche das positive Image Genfs prägen. Zusätzliche Vorteile sind nach Auffassung der INRO die hohe Lebensqualität in „Genf“, welche die Region für Mitarbeiter aus dem Ausland attraktiv macht, sowie der kürzliche Beitritt der Schweiz als vollwertiges Mitglied zur UNO.

Unter den Nachteilen der Niederlassung in „Genf“ nennen die INRO an erster Stelle die hohen Lebenshaltungskosten und folglich die relativ hohen Gehälter, die den Mitarbeitenden gezahlt werden müssen und die den Haushalt der kleinen INRO belasten. Die Distanz zum Sitz und die Probleme bei der Erteilung von Arbeitsbewilligungen für Angestellte (besonders für Mitarbeiter aus

14 Fernando Henrique Cardoso, High Level Panel on UN-Civil Society: Civil Society and Global Governance. Contextual Paper, New York, Organisation der Vereinten Nationen, Juni 2003, 11 S.

15 Rede von Fernando Henrique Cardoso, Präsident des hochrangigen Panels über das Verhältnis zwischen der UNO und der Zivilgesellschaft anlässlich der NRO-Jahreskonferenz des Department of Public Information der UNO, New York, 8. September 2003.

16 Der Panelbericht wurde Ende Juni veröffentlicht. Nähere Informationen dazu unter: <www.un.org/ reform/panel.htm>. 
Entwicklungsländern) werden von einigen INRO ebenfalls unter den Nachteilen aufgeführt.

Angesichts der Stellungnahmen der INRO scheinen jedoch insgesamt die Vorteile des Standorts „Genf“ gegenüber den Nachteilen deutlich zu überwiegen. Dieses Fazit ist indessen mit Vorsicht zu geniessen: Die Rahmenbedingungen verändern sich möglicherweise, und die Beziehungen bzw. die Interaktionen zwischen Bund und INRO müssen an die wechselhaften Gegebenheiten des internationalen Genfs angepasst werden. Nicht $\mathrm{zu}$ vergessen ist ausserdem die direkte Konkurrenz anderer europäischer Städte wie Bonn, Lyon und Brüssel ${ }^{17}$.

Schliesslich ist auch der grosse Beitrag der INRO selbst, ihres Personals und ihrer Delegierten hervorzuheben, welche zusammen mit den verschiedenen internationalen Organisationen der Rhonestadt ein echtes internationales Profil verleihen. Die damit verbundene kulturelle und sprachliche Vielfalt ergänzt das bedeutende ökonomische Gewicht der INRO.

\section{Schlussfolgerungen}

Ziel dieser Zusammenfassung war es, einige Beobachtungen zur Welt der internationalen NRO in "Genf“ vorzustellen und als ersten Schritt zum Verständnis ein klärendes Licht auf diesen relativ undurchsichtigen, sich stetig wandelnden Kosmos zu werfen. Wie die Studie zeigte, arbeiten viele offizielle und nichtstaatliche Organisationen mit den INRO zusammen und besitzen verschiedenste Informationen über den Sektor. Daher ist es bedauerlich, dass die verschiedenen Strukturen ihre Informationen nicht besser koordinieren und teilen. Sofern dem Bund wirklich daran liegt, eine fundierte und zweckmässige Politik für die INRO zu gestalten, sollte er im ureigenen Interesse einen besseren Überblick anstreben. Dazu ist eine Optimierung der Informationsverbreitung und -koordinierung unabdingbar.

Über den deskriptiven Ansatz hinaus ist festzustellen, dass den Behörden für die Beziehungen mit den INRO verschiedene Optionen zur Wahl stehen. Gemäss der oben dargelegten Bestandsaufnahme sollte die Unterstützung der schweizerischen Behörden an die internationalen Nichtregierungsorganisationen in drei Bereichen verstärkt werden :

1) Förderung von Entwicklung und Wachstum eines oder mehrerer Exzellenzpole wie Frieden, Menschenrechte oder autochthone Völker (siehe oben);

2) verstärkte Unterstützung in den Bereichen Empfang und Förderung der Arbeit der INRO über vorhandene (staatliche oder nichtstaatliche) Strukturen, denen aber die Ressourcen fehlen, bzw. die schlecht koordiniert sind;

3) Verbesserung der Rahmenbedingungen für alle INRO und ihr Personal, welche das Steuerwesen, die Förderung der internationalen Schulen sowie die

17 Erwähnenswert ist das Beispiel der Internationalen Union für die Erhaltung der Natur (International Union for the Conservation of Nature, IUCN): Die IUCN hatte bereits den Umzug des Sitzes nach Bonn in die Wege geleitet, als in Bern die Alarmglocke schrillte und man - etwas spät - feststellte, dass Bern günstigere Bedingungen als die Konkurrenten anbieten musste, um die Organisation in der Schweiz zu halten. Schliesslich erhielt die IUCN (wie im Übrigen auch vier weitere grosse NRO) den mit grosszügigeren Privilegien verbundenen Status einer „quasi-staatlichen“ Organisation, so dass sie schliesslich auf den geplanten Umzug verzichtete. 
Gewährung von Arbeitsbewilligungen für Ehepartner von INRO-Mitarbeitenden betreffen.

Im aktuellen politischen Kontext werden sich indessen die Budgetkürzungen in der Entwicklungszusammenarbeit, im sozialen Bereich und in der internationalen Solidarität zwangsläufig in noch knapperen Ressourcen für die INRO niederschlagen ${ }^{18}$. Angesichts der gegenwärtigen Konjunktur können die Behörden die Unterstützung für die INRO nicht aufstocken, was zu Lasten einer längst überfälligen kohärenten und koordinierten Förderpolitik für das nichtstaatliche internationale Genf geht.

\section{BIBLIOGRAPHIE}

Annan Kofi, Stärkung der Vereinten Nationen: Eine Agenda für weitere Veränderungen. Bericht an die Generalversammlung, A/57/387, 9. September 2002, 38 S.

Baccaro Lucio, Civil Society, NGOs, and Decent Work Policies: Sorting out the Issues, ILO Discussion Papers DP/127/2001, 2001, 34 S.

Cardoso Fernando Henrique, High Level Panel on UN-Civil Society: Civil Society and Global Governance. Contextual Paper, New York, Organisation der Vereinten Nationen, Juni 2003, 11 S.

Entwicklungszentrum der Organisation für wirtschaftliche Zusammenarbeit und Entwicklung (OECD), Répertoire des organisations non gouvernementales actives dans le domaine du développement durable, Band I: Europe, Paris, 1996, 765 S.

Staatskanzlei, Annuaire officiel de la République et Canton de Genève 2003, Genf, Dezember 2002, $479 \mathrm{~S}$.

Bundeskanzlei, Der Bund kurz erklärt 2003, Bern, Februar 2003, 80 S.

Chopard Jean-Luc, Genève et les organisations internationales non gouvernementales, Genf, Februar 2002, $7 \mathrm{~S}$.

Wirtschafts- und Sozialrat der Vereinten Nationen (ECOSOC), Résolution 1296 de l'ECOSOC concernant les dispositions relatives aux consultations avec les ONG, 1968.

Coopération internationale pour la démocratie, Les ONG dans la restructuration des Nations unies, dossier, Coopération internationale pour la démocratie, Nr. 5, Paris, IATP; IBASE; INRA; SOLAGRAL, 1995, S. 157-173.

Deutsche Stiftung für Internationale Entwicklung, Thesaurus für wirtschaftliche und soziale Entwicklung (TWSE), 1999.

Doucin Michel, Guide de la liberté associative dans le monde. Les législations des sociétés civiles de 138 pays, Paris, Haut Conseil de la coopération internationale, 2000, 572 S.

Eco'Diagnostic, Vereinte Nationen, International Geneva Yearbook 2002-2003: Organization and Activities of International Institutions in Geneva, Band 16, Genf, 2002, 608 S.

Florinetti Sophie, Genève a-t-elle les moyens de sa vocation de ville internationale?, Fondation pour Genève, April 2001, 29 S.

Forum mondial de la société civile, Renforcer la coopération internationale, Genf, Juli 2003, 88 S.

Laxton Valérie, L'impact économique de l'activité de la Genève internationale. Le rôle des organisations non gouvernementales (ONG), Genf, Mandat International, Juni 1999, 23 S.

Observatoire de la Fondation pour Genève, Genève a-t-elle les moyens de sa vocation de ville internationale?, Genf, Februar 2000, 15 S.

Office cantonal de la statistique, Les organisations internationales à Genève et en Suisse. Résultats de l'enquête 2002, Genf, Februar 2003, 23 S.

Bundesamt für Umwelt, Wald und Landschaft (BUWAL), Geneva!, Bern, Juni 2002.

—, ,Genf - eine Denkfabrik der globalen Umweltpolitik“, Umweltschutz, Nr. 3, März 1999, S. 59.

Organisation der Vereinten Nationen, UN System and Civil Society: An Inventory and Analysis of Practices, Background paper for the Secretary-General's Panel of Eminent Persons on UN relations with Civil Society, New York, Mai 2003, 27 S.

18 Im Kanton Genf wurde dieser Schritt bereits getan, während in Bern bestimmte politische Formationen Druck auf die Bundesverwaltung ausüben, ihre Entscheidungen zur Förderung bestimmter INRO zu begründen, und sich offen dafür aussprechen, die Mittel an das internationale Genf zu kürzen. 
Umweltprogramm der Vereinten Nationen (UNEP), Genève verte: le guide, Genf, September 2001, $86 \mathrm{~S}$. Republik und Kanton Genf, Le Classeur du protocole. Répertoire des autorités genevoises et fédérales, des organisations internationales et non gouvernementales établies à Genève, Genf, Januar 2003.

Sottas Eric, ONG et droits de l'homme: pour des stratégies cohérentes, Weltorganisation gegen Folter, November 2000, $31 \mathrm{~S}$.

Topçu Yasemin, Humanitarian NGO-networks : Identifying Powerful Political Actors in an International Policy Field, Wissenschaftszentrum Berlin für Sozialforschung, Januar 1999, 42 S.

United Nations Non-Governmental Liaison Service, United Nations System: A Guide for NGOs, 10. Auflage, Genf; New York, Januar 2003, 242 S.

Weiss G. Thomas, Gordenker Leon, NGOs, The UN, and Global Governance, Boulder, Colorado, Lynne Rienner, 1996, S. 7-47.

Willets Peter, „What Is a Non-Governemental Organization?“, UNESCO Encyclopedia of Life Support Systems, 2002, S. 1-12.

Ziegler Sébastien, Mémorandum pour une politique fédérale active en faveur du secteur international non gouvernemental, Genf, Mandat International, Juli 2000, 17 S. 